\title{
Generalized System of Mono- and Bi-Propellant Propulsion for Microsatellite
}

\author{
By Hironori SAHARA and Tatsuya IDE \\ Department of Aerospace Engineering, Tokyo Metropolitan University, Tokyo, Japan
}

(Received July 14th, 2009)

The authors completed those propulsion system for microsatellite based on the SAFETY FIRST policy and EFFECTIVE COTS, by the beginning of 2008, which can work as both mono- and bi-propellant propulsion system and attain wide-range impulse bit and total impulse by switching the mono- and bi-propellant modes. We have been devoting ourselves to develop an upgraded version of the propulsion system since 2008. We reviewed and introduced an improved catalyst and self pressurization propellant combination, and re-designed the propulsion diagram. As the result, the propulsion system can be simpler and lighter than the previous one because the number of elements in the diagram is reducible. This will be a breakthrough for microsatellite to install a propulsion system on it.

Key Words: Propulsion, Monopropellant, Bipropellant, Microsatellite, Hydrogen Peroxide

\section{Introduction}

Microsatellites developed by non-governmental association or university groups are now in vogue not only in Japan ${ }^{1,2)}$ but also in the world, as typified by SOHLA-1 and SOHLA-2 microsatellites developed by the Space Oriented Higashi-osaka Leading Association (SOHLA). However, they tend to be far from installing propulsion so far due to its high cost, and the conventional propulsion is awfully expensive since it is developed as a unique or identifiable product by proper specialists with quite high reliability. But if with a propulsion on microsatellite, space utilization or observation will be popularized for the more developers or observers, or even regular people. Here, propulsion of moderate price and decent performance is eagerly desired and it will broaden out the application of microsatellites and contribute to the development and prosperity of humankind.

\section{Previous Attainment by Author}

The author started to develop such a propulsion system for microsatellite in 2004 as a part of the SOHLA-2 project, and completed it, based on the SAFETY FIRST policy and EFFECTIVE COTS, by the beginning of 2008. ${ }^{3)}$ It depends on the following design concept: First, microsatellite generally has relatively short lifetime as 3 months or so and the propulsion should work to acquire necessary thrust or delta- $\mathrm{V}$ in the operation period. Second, supplying electricity on the microsatellite is not so large due to its small volume or surface areas. Therefore, the propulsion should attain large thrust density suggesting that chemical propulsion is the most suitable. It can work as both mono- and bi-propellant propulsion system and attain wide-range impulse bit and total impulse by switching the mono- $(<1 \mathrm{~N}, 60 \mathrm{sec}$ of Isp) and bi-propellant ( $>1 \mathrm{~N}, 120-150 \mathrm{sec}$ of Isp) modes. Figures 1 and 2 show its assembly installed in the SOHLA-2 structure and picture under its captive test.

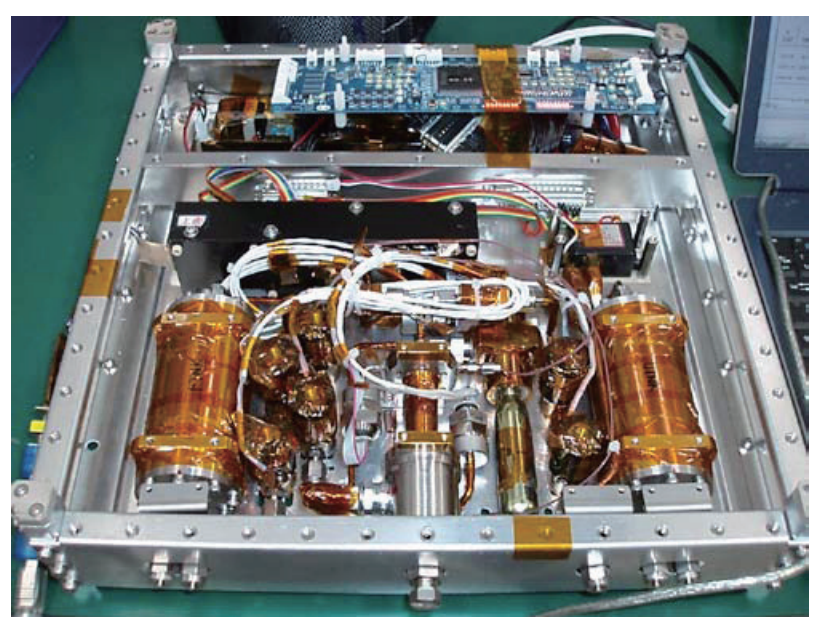

Fig. 1. Propulsion system for SOHLA-2.

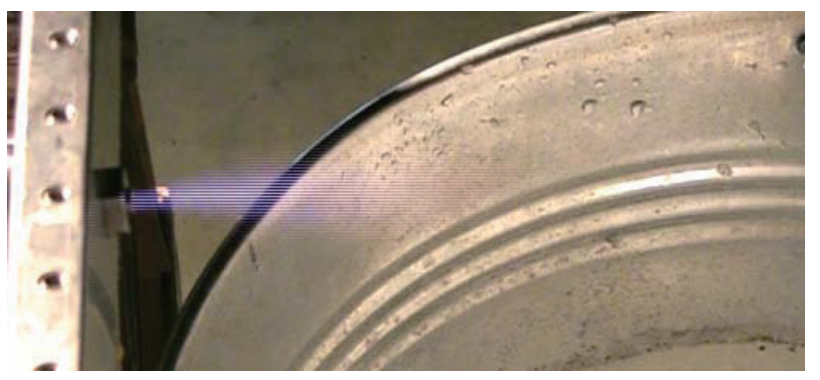

Fig. 2. Captive test in bi-propellant mode (without nozzle).

\section{Further Improvement}

\subsection{Diagram}

We have been devoting ourselves to develop an upgraded version of the above propulsion system since 2008. We reviewed and introduced an improved catalyst and self pressurization propellant combination, and re-designed the propulsion diagram as shown in Figs. 3. As the result, the 
propulsion system can be simpler and lighter than that for SOHLA-2 because the number of elements in the diagram is reducible by abbreviating the pressuring line (G-Line) to remain oxidizer line (O-Line) and fuel line (F-Line). This will be a breakthrough for microsatellite to install a propulsion system on it. Figure 4 shows its experimental setup assembled.
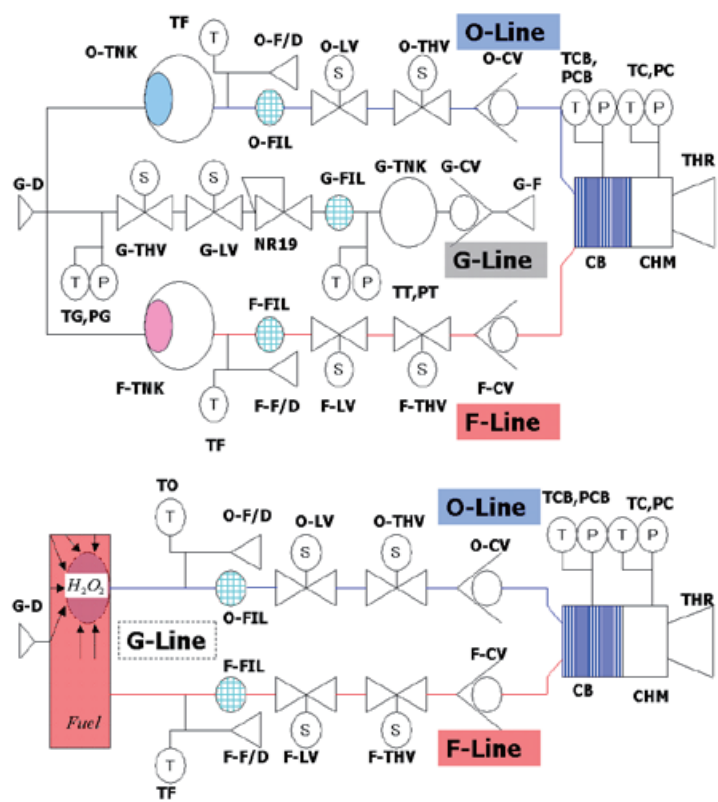

Figs. 3. Diagram of propulsion systems: upper) for SOHLA-2, lower) re-desgined.

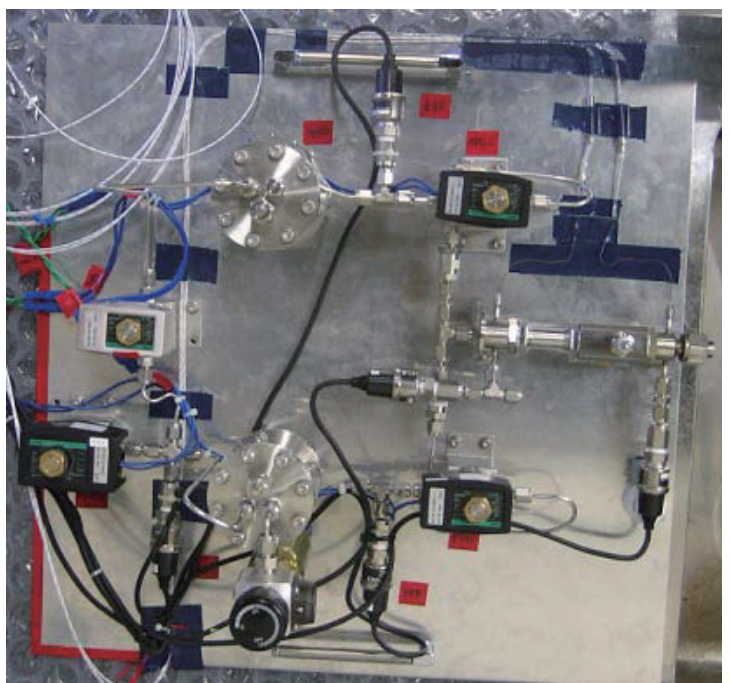

Fig. 4. Experimental setup of the re-designed propulsion system.

\subsection{Fuel}

We selected Dimethyl Ether (DME) as a fuel in the propulsion system. DME has the chemical structural formula shown in Fig. 5. DME has 6 atm of vapor pressure around room temperature, wide explosive range, and high cetane number compared with the other fuels as shown in Fig. 6 and Table 1. Especially, the vapor pressure is very suitable for a thruster with combustion pressure of $4 \mathrm{~atm}$ designed, related later.<smiles></smiles>

Fig. 5. Chemical structural formula of DME.

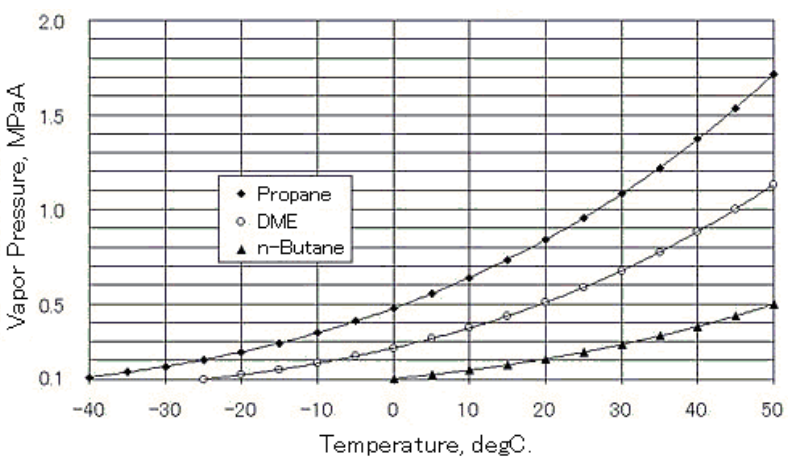

Fig. 6. Vapor pressure of DME and the other fuels. ${ }^{4)}$

Table 1. Properties of DME and the other fuels. ${ }^{4}$

\begin{tabular}{|c|c|c|c|c|c|c|}
\hline & DME & Propane & Methane & Methanol & LGO \\
\hline \multicolumn{2}{|c|}{ Chemical Formula } & $\mathrm{CH}_{3} \mathrm{OCH}_{3}$ & $\mathrm{C}_{3} \mathrm{H}_{8}$ & $\mathrm{CH}_{4}$ & $\mathrm{CH}_{3} \mathrm{OH}$ & \\
\hline \multicolumn{2}{|c|}{ Boiling Point, degC. } & -25 & -42 & -161.5 & 64.6 & $180-370$ \\
\hline \multicolumn{2}{|c|}{ Liquid Density, $\mathrm{g} / \mathrm{cm}^{3}$} & 0.67 & 0.49 & & 0.79 & 0.84 \\
\hline \multicolumn{2}{|c|}{ Gas Specific Gravity } & 1.59 & 1.52 & 0.55 & & \\
\hline \multicolumn{2}{|c|}{ Vapor Pressure, MPa } & 0.61 & 0.94 & 24.9 & & \\
\hline \multicolumn{2}{|c|}{ Explosive Range, \% } & $3.4-27$ & $2.1-9.5$ & $5.0-15.0$ & $5.5-36.0$ & $0.6-6.5$ \\
\hline \multicolumn{2}{|c|}{ Cetane Number } & $55-60$ & 5 & 0 & 5 & $40-55$ \\
\hline \multirow{2}{*}{ LHV } & $\left(\mathrm{kJ} / \mathrm{Nm}^{3}\right)$ & 59,400 & 91,300 & 36,000 & & \\
\hline & $(\mathrm{kJ} / \mathrm{kg})$ & 28,900 & 46,500 & 50,200 & 20,100 & 41,900 \\
\hline
\end{tabular}

\subsection{Thruster}

A thruster should be available to have capability of working in both mono-propellant mode and bi-propellant mode. In addition, we intended to examine various catalyst and nozzle ratio in the thruster, so that we designed a splittable thruster composed of those 4 parts as shown in Fig. 7, which consists of Injector Part, Catalyst Bed, Combustion Chamber, Nozzle.

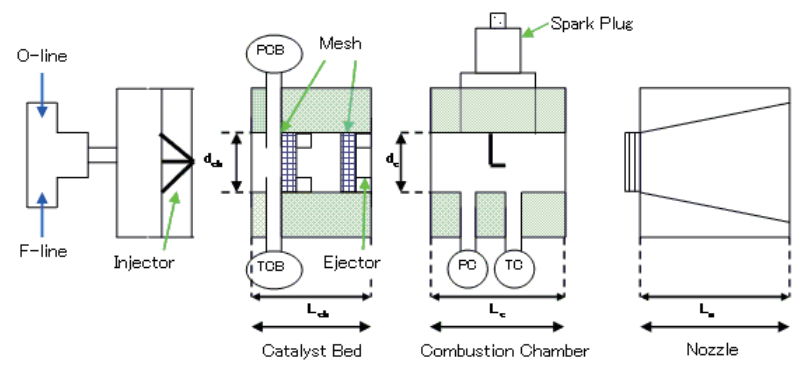

Fig. 7. A splittable thruster.

\subsection{Injector part}

Propellant, especially Hydrogen Peroxide should be 
atomized at an injector in order to realize high efficiency of its decomposition in the catalyst bed. We adopted an impingement type injector which had 3 small holes tilted as shown in Fig. 8. A shower type ejector shown in Fig. 9 which aligns the propellant flow perturbed by the granulated catalyst, was set just after the catalyst bed.

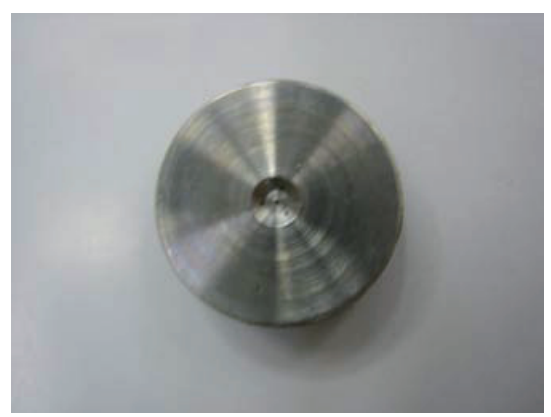

Fig. 8. Impingement type injector.

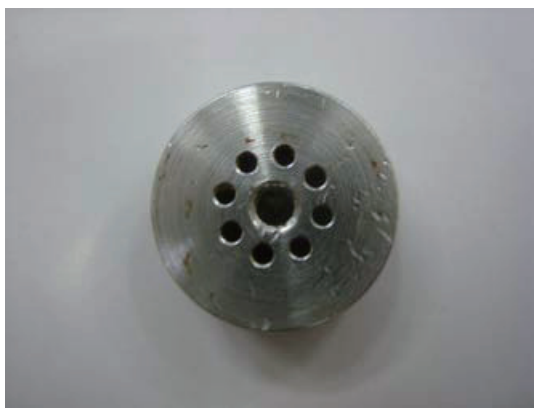

Fig. 9. Shower type ejector.

\subsection{Catalyst bed}

In the catalyst bed shown in Fig. 10, one of the propellants, Hydrogen Peroxide, should be efficiently decomposed by a suitable catalyst. We found such a catalysts, called Catalyst-S and Catalyst-K, which were granulated type catalysts with particle size of \#14-18 and \#25-30, respectively, as shown in Fig. 11. One of the two catalysts is loaded into the catalyst bed and a pair of \#14-18 mesh shown in Fig. 11 is set at entrance and exit of the catalyst bed in order to avoid the granulated catalyst from leakage. Inner diameter of the catalyst bed is matched to that of combustion chamber to maintain smooth propellant flow from the catalyst bed to the combustion chamber.

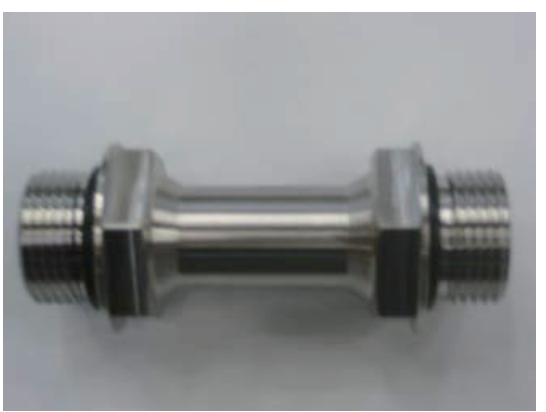

Fig. 10. Appearance of Catalyst Bed.

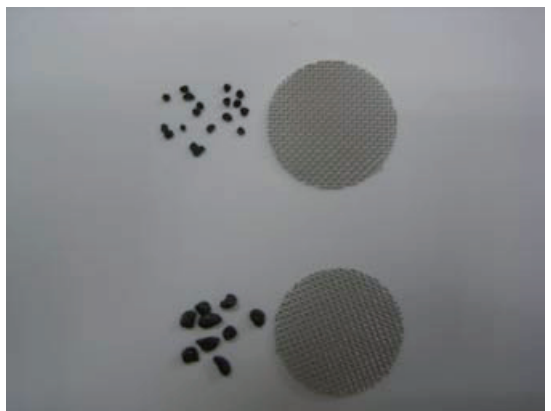

Fig. 11. Catalysts and a pair of mesh.

\subsection{Combustion chamber}

In order to design combustion chamber, we first calculated and estimated combustion in the combustion chamber with Chemical Equilibrium with Application (CEA). Supposing that DME in a propellant tank has $6 \mathrm{~atm}$ of vapor pressure, 4.4 atm is probable in the combustion chamber considering pressure drop at valves and the injector, under that propellants are Hydrogen Peroxide of $60 \mathrm{wt} \%$ as oxidizer and DME as fuel and nozzle area ratio is 50 . As the result, we obtained an estimation shown in Table 2.

Table 2. Estimation in combustion chamber.
\begin{tabular}{|l|c|c|c|}
\hline Item & - & Value & Unit \\
\hline \hline Density in Combustion Chamber & $\rho_{c}$ & 0.6506 & $\mathrm{~kg} / \mathrm{m}^{3}$ \\
\hline Specific Exhaust Velocity & $c^{*}$ & 1277.3 & $\mathrm{~m} / \mathrm{s}$ \\
\hline Thrust Coefficient & $C_{F}$ & 1.81 & - \\
\hline Specific Impulse & $I_{s p}$ & 234.5 & $\mathrm{sec}$ \\
\hline
\end{tabular}

By using the above values, we determined diameter and length of the combustion chamber. When thrust, $F$, is set at $1 \mathrm{~N}$,

$$
\Delta m=\frac{F}{g I_{s p}}=4.35 \times 10^{-4}
$$

Here,

$$
P=\rho R T=\frac{m R T}{V}
$$

We approximately replaced $m$ by $\Delta m$, and obtained specific volume of combustion chamber, $V_{c}^{*}$, as follows:

$$
V_{c}^{*}=\frac{\Delta m}{\rho}=\frac{4.35 \times 10^{-4}}{6.506 \times 10^{-1}}=6.68 \times 10^{-4}
$$

Considering stay time in combustion chamber as $t_{s}=0.011 \mathrm{sec}$, volume of combustion chamber, $V_{c}$, is obtained as follows:

$$
V_{c}=6.68 \times 10^{-4} \times 0.011 \cong 7.35 \times 10^{-6}
$$

Ratio of length and diameter of combustion chamber, $L_{c} / d_{c}$, is $2-4$ in general. Here, we determined $L_{c} / d_{c}=4$ and finally $d_{c}=13.3 \mathrm{~mm}$ and $L_{c}=53.2 \mathrm{~mm}$, shown in Fig. 12 . 


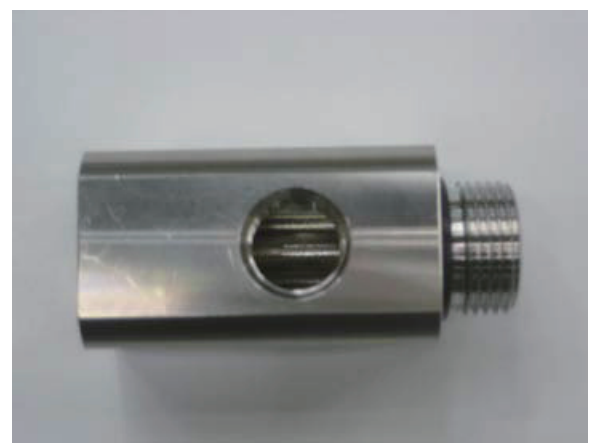

Fig. 12. Combustion Chamber (without Spark Plug).

We conducted strength calculation with the above values. Since radial thickness of the combustion chamber, $t$, is $2.35 \mathrm{~mm}$ and the ratio of $t$ and $d_{c} / 2$ is 0.35 , the combustion chamber is regarded as thick cylinder. Its material is SUS304 having tension strength of $\sigma_{B}=520 \mathrm{~N} / \mathrm{mm}^{2}$, and maximum values of radial stress, $\sigma_{r \max }$, circumferential stress, $\sigma_{\theta \max }$ axial stress, $\sigma_{Z \max }$, are obtained as follows:

$$
\begin{aligned}
& \sigma_{r \max }=P \frac{1-(b / a)^{2}}{(b / a)^{2}-1}=0.446 \times \frac{1-(9 / 6.65)^{2}}{(9 / 6.65)^{2}-1}=-0.446 \\
& \sigma_{\theta \max }=P \frac{1+(b / a)^{2}}{(b / a)^{2}-1}=0.446 \times \frac{1+(9 / 6.65)^{2}}{(9 / 6.65)^{2}-1}=1.519 \\
& \sigma_{Z \max }=P \frac{1}{(b / a)^{2}-1}=0.446 \times \frac{1}{(9 / 6.65)^{2}-1}=0.536
\end{aligned}
$$

where $a=d_{c} / 2=6.65 \mathrm{~mm}$ and $b=9 \mathrm{~mm}$ are inner and outer radii of the combustion chamber. Therefore, the maximum stress is, $\sigma_{\theta \max }$ and factor of safety, $S$, is calculated as

$$
S=\frac{\sigma_{B}}{\sigma_{\theta \max }}=\frac{520}{1.519}=342
$$

which is sufficiently large value.

\subsection{Nozzle}

Throat area, $A_{t}$, and throat diameter, $d_{t}$, are obtained as

$$
\begin{aligned}
& A_{t}=\frac{V_{c}}{L^{*}}=8.91 \times 10^{-6}=\frac{\pi d_{t}^{2}}{4} \\
& \rightarrow d_{t}=\sqrt{A_{t} \times 4 / \pi}=1.68 \times 10^{-3}
\end{aligned}
$$

Since nozzle area ratio, $A_{e} / A_{t}$, is fixed at 50 in this propulsion system, nozzle exit area and diameter, $A_{e}$ and $d_{e}$, are obtained:

$$
\begin{aligned}
& A_{e}=4.45 \times 10^{-4} \\
& \rightarrow d_{e}=\sqrt{A_{e} \times 4 / \pi}=11.9 \times 10^{-3}
\end{aligned}
$$

Here, half apex angle is 15 degrees, nozzle length, $L_{n}$, is obtained as follows:

$$
L_{n}=\frac{d_{e} / 2-d_{t} / 2}{\tan 15^{\circ}}=\frac{5.11 \times 10^{-3}}{\tan 15^{\circ}}=19.1 \times 10^{-3}
$$

Considering processing accuracy and manufacturing cost, we finally determined dimensions of $d_{t}=1.41 \mathrm{~mm}, d_{e}=10.0 \mathrm{~mm}$, and $L_{n}=16.0 \mathrm{~mm}$, respectively, as shown in Fig. 13 .

Thus, we realized a re-designed thruster shown in Fig. 14.

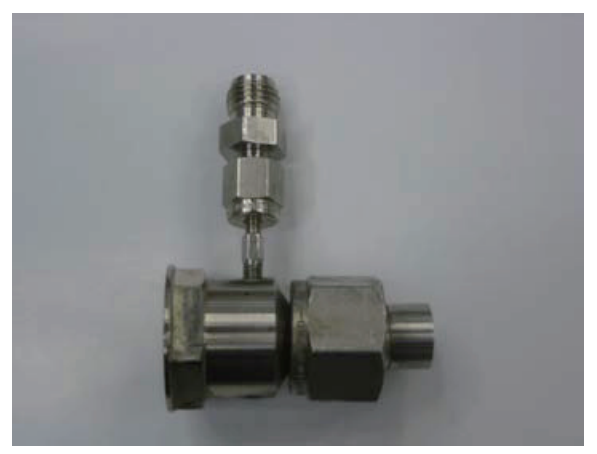

Fig. 13. Nozzle (with a measuring port).

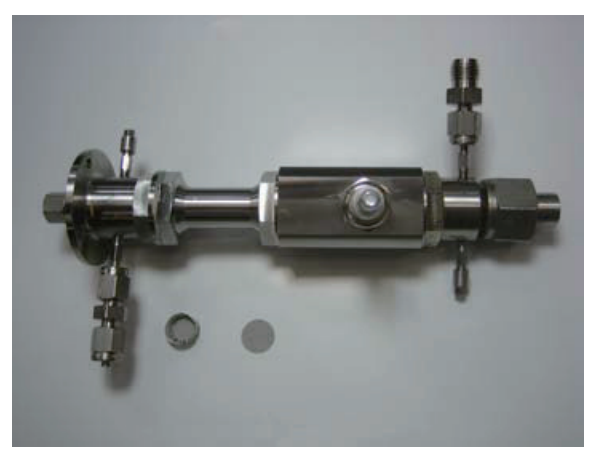

Fig. 14. Thruster assembled (with Spark Plug and measuring ports).

\section{Captive Test Results}

We conducted captive tests by using the experimental setup of the re-designed propulsion system and the thruster. By switching electromagnetic valves in the O-line and the F-line, it works as 4 modes as follows.

\subsection{Regulated mono-propellant mode}

We conducted captive test of regulated mono-propellant mode with Hydrogen Peroxide as propellant. Propellant optionally pressured by regulated nitrogen gas at $5 \mathrm{~atm}$ from G-D port in Fig. 3, is fed to the thruster. It is decomposed in the catalyst bed to oxygen gas and mixture of gas and vapor of water. Its temperature and pressure in the combustion chamber rapidly rose just after starting injection due to decomposition heating up to boiling point and $4.4 \mathrm{~atm}$ as shown in Fig. 15, which goes just as designed. We tested both catalyst-K (\#1, $\# 2$ ) and catalyst-S (\#3, \#4), and varied duty ratio of feeding electromagnetic valves as $100 \%(\# 1, \# 3)$ and $50 \%(\# 2, \# 4)$. As the result, we obtained adequate thrust performances for microsatellite as shown in Table 3.

\subsection{Mono-propellant mode pressured by DME}

We conducted captive test of mono-propellant mode with Hydrogen Peroxide as propellant, which was pressured by vapor pressure of DME. We were supposed that the pressure should be $6 \mathrm{~atm}$ at room temperature, but this test was done in winter. Since ambient temperature was around $10 \mathrm{degC}$., its vapor pressure was only $3 \mathrm{~atm}$. As the result, pressures in the catalyst bed and combustion chamber were about $200 \mathrm{kPa}$ and 
$160 \mathrm{kPa}$, respectively, as shown in Fig. 16. However, we estimated thrust performance with the result and over $50 \mathrm{sec}$ of specific impulse was obtained as shown in Table 4.

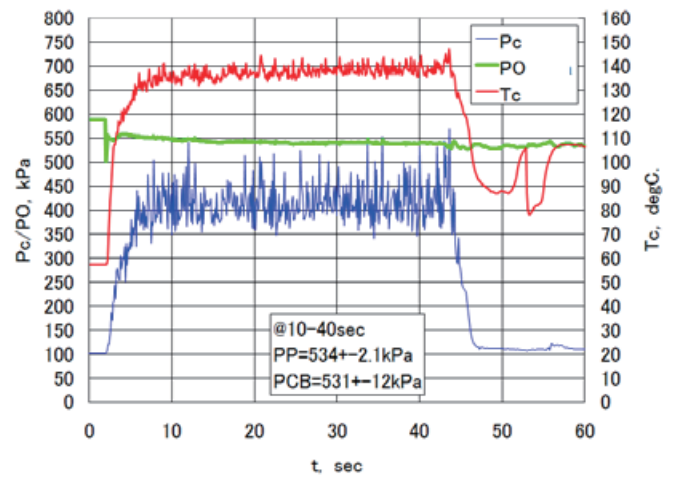

Fig. 15. A captive test result of regulated mono-propellant mode with Hydrogen Peroxide.

Table 3. Thrust performances of various catalysts and duty ratio.

\begin{tabular}{|l||c|c|c|c|c|c|}
\hline Thrust Performances & - & Unit & $\mathbf{\# 1}$ & $\mathbf{\# 2}$ & $\mathbf{\# 3}$ & $\mathbf{\# 4}$ \\
\hline \hline Mass Flow Rate & $\Delta m$ & $\mathrm{~kg} / \mathrm{s}$ & 1.51 & 1.28 & 1.46 & 1.38 \\
\hline Thrust Coefficient & $C_{F}$ & - & 1.80 & 1.80 & 1.80 & 1.80 \\
\hline Specific Exhaust Velocity & $c^{*}$ & $\mathrm{~m} / \mathrm{s}$ & 415 & 436 & 403 & 410 \\
\hline Thrust & $F$ & $\mathrm{~N}$ & 0.82 & 0.81 & 0.85 & 0.82 \\
\hline Specific Impulse & $I_{s p}$ & $\mathrm{sec}$ & 55.0 & 64.1 & 59.2 & 60.3 \\
\hline
\end{tabular}

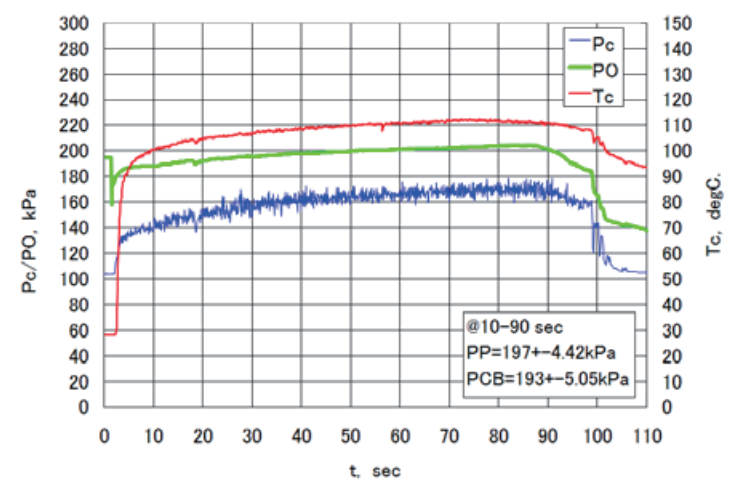

Fig. 16. A captive test result of mono-propellant mode with Hydrogen Peroxide pressured by DME in winter.

Table 4. Thrust performances pressured by DME.

\begin{tabular}{|l||c|c|c|}
\hline Thrust Performances & - & Unit & - \\
\hline \hline Mass Flow Rate & $\Delta m$ & $\mathrm{~kg} / \mathrm{s}$ & 0.64 \\
\hline Thrust Coefficient & $C_{F}$ & - & 1.80 \\
\hline Specific Exhaust Velocity & $c^{*}$ & $\mathrm{~m} / \mathrm{s}$ & 358 \\
\hline Thrust & $F$ & $\mathrm{~N}$ & 0.330 \\
\hline Specific Impulse & $I_{s p}$ & $\mathrm{sec}$ & 52.6 \\
\hline
\end{tabular}

\subsection{Mono-propellant mode of DME}

We conducted captive test of mono-propellant mode with DME as propellant. DME was fed to the thruster by self pressurization. The pressure was just vapor pressure of DME itself, but the test was conducted in the same winter so that the vapor pressure was only $200 \mathrm{kPa}$ as shown in Fig. 17 . Temperature dropped to $-20 \mathrm{degC}$., the boiling point of DME, and pressure in the combustion chamber rose to $140 \mathrm{kPa}$.

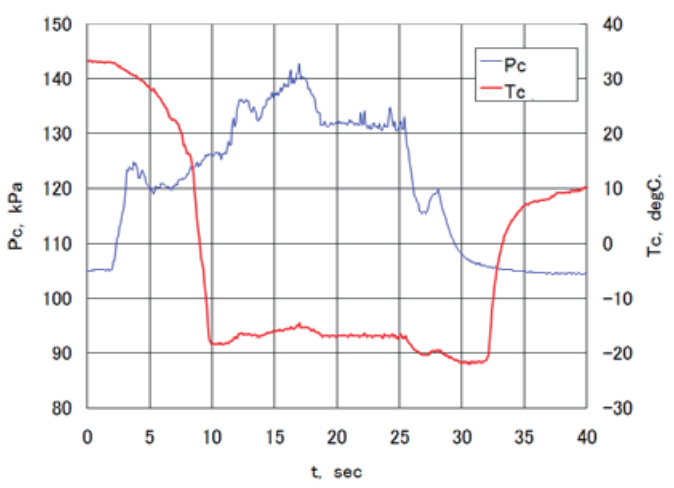

Fig. 17. A captive test result of mono-propellant mode with DME.

In fact there is mixture of gas and vapor of DME in the combustion chamber, but we estimated thrust performance supposing it as approximate ideal gas. Specific impulse is calculated with the chamber and ambient pressures,

$$
I_{s p}=\frac{\sqrt{\frac{2 k}{k-1} \cdot \frac{\bar{R} T_{c}}{M_{F}}\left(1-\frac{P_{e}}{P_{c}}\right)^{\frac{k-1}{k}}}}{g} \cdot \eta_{c f}
$$

and $51.7 \mathrm{sec}$ of specific impulse was obtained.

\subsection{Bi-propellant mode}

We conducted ignition test of bi-propellant mode. Propellants were Hydrogen Peroxide for oxidizer and DME for fuel. Electromagnetic valves in both the O-line and the F-line were simultaneously opened, and Spark Plug started its discharge in the combustion chamber. However, no ignition occurred. Now we are investigating its reason and trying to improve condition of the ignition. We estimate that liquid droplet of mixture of DME and water derived from decomposition of Hydrogen Peroxide is too large and spark energy is too small to gasify and be ignited. It suggests that atomization just after the catalyst bed, that is, at the upper region of the combustion chamber is quite necessary, which indicates configuration of the ejector should be improved.

\section{Conclusion}

We re-designed the propulsion system for microsatellite by effective self pressurization of propellant which has adequate vapor pressure, and manufactured an experimental setup of generalized system of mono- and bi-propellant propulsion using Hydrogen Peroxide and Dimethyl Ether as propellants. We were supposed that it worked both mono- and bi-propellant mode by switching electromagnetic valves as appropriate. As the result, mono-propellant mode attained favorable thrust performances such as specific impulse of over 50 - 60 seconds, especially mono-propellant mode with Hydrogen Peroxide virtually reached its perfection. And we obtained two issues: the first is to maintain temperature of 
DME having sufficient vapor pressure, and the second is to improve the ejector configuration so that sufficient evaporation and ignition occurs in the combustion chamber and the propulsion system works also as bi-propellant mode.

\section{References}

1) Nakamura, Y.: University of Tokyo's Ongoing Student-Lead Pico-Satellite Projects - CubeSat XI and PRISM, Proceedings of 55th International Astronautical Congress, IAF,
IAC-04-IAA.4.11.4.06, 2004.

2) Kuwahara, T.: Kyushu University Micro-Satellite QTEX Project, Proceedings of 55th International Astronautical Congress, IAF, IAC-04-IAA.4.11.5.06, 2004.

3) Sahara, H., Nakasuka, S., Kobayashi, C.: Generalized Propulsion System for Microsatellite Based on Hydrogen Peroxide, Trans. JSASS Space Tech. Japan, 7, ISTS26 (2009).

4) http://www.khk.or.jp/activities/research_development/lpg_lab/faq_d me.html [cited on January 31, 2009]. 